\title{
Biometrics: Different Approaches for Using Gaussian Mixture Models in Handwriting
}

\author{
Sascha Schimke ${ }^{1}$, Athanasios Valsamakis ${ }^{2}$, Claus Vielhauer ${ }^{1}$, and \\ Yannis Stylianou ${ }^{2}$ \\ 1 Otto-von-Guericke University of Magdeburg, \\ 2 University of Crete
}

\begin{abstract}
In this work in progress paper we discuss an established as well as a new approache to the use of Gaussian Mixture Models (GMMs) for handwriting biometrics. The technique of GMMs is well explored for the domain of speech processing and we evaluate ways to use them for handwriting biometrics, too.
\end{abstract}

\section{Introduction}

At present, certain biometric traits are very popular in the public discussion. Mainly these are fingerprint, face or iris recognition for the purpose of verification or identification. In our work we focus on the active trait of on-line handwriting biometrics and in particular signature identification. Most people are familiar with handwritten signature because of regularly usage for example to affirm contracts or sign forms.

In the following, we elaboarte on new approaches derived from speaker recognition and theoretically outline their adaptivity towards signature authentication.

\section{Gaussian Mixture Models (GMMs)}

Gaussian mixture models have been successfully used for speaker recognition tasks [2]. Here we suggest to use GMMs for signature recognition applications. The main idea is to represent the biometric features as the probability density function of one signee, with a mixture of Gaussians.

A Gaussin mixture density function is primarily a weighted sum of $M$ constituent functions, and in notational form

$$
p(\mathbf{x} \mid \lambda)=\sum_{i=1}^{M} a_{i} f_{i}(\mathbf{x})
$$

where $\mathbf{x}$ is a $D$-dimensional random vector, $f_{i}(\mathbf{x}), i=1,2 \cdots M$ are the constituent Gaussian density functions, and $a_{i}$ are the mixture weights. Each constituent Gaussian density is a $D$-variate normal density function. For the GMM 
function $p(\mathbf{x} \mid \lambda)$ to be a legitimate probability density function the constraint $\sum_{i=1}^{M} a_{i}=1$ must be satisfied.

Each signee has its own density function, that is $p\left(\underline{x} \mid \lambda_{s}\right)$ where $\lambda_{1}, \lambda_{2} \cdots \lambda_{S}$ are the different signees. The parameters of signee $s$ 's density are denoted as $\left\{a_{i}, m_{i}, \Sigma_{i}\right\}, i=1,2 \cdots M$. For identification, a maximum likelihood classifier is used. For a number of signees $S$ represented by $\lambda_{1}, \lambda_{2} \cdots \lambda_{S}$ our goal is to find the signee with maximum posterior probability for the feature vectors $X=$ $\left\{x_{1}, x_{2} \cdots x_{T}\right\}$ extracted by the signature to be tested. The probability of being signee $\lambda_{s}$ given the feature vectors of the testing signature is given by the Bayes' formula

$$
\operatorname{Pr}\left(\lambda_{s} \mid X\right)=\frac{p\left(X \mid \lambda_{s}\right) \operatorname{Pr}\left(\lambda_{s}\right)}{p(X)}
$$

We can assume without loss of generality equal prior probabilities $\operatorname{Pr}\left(\lambda_{s}\right)$ for all speakers. The denominator can be ignored as being a constant for all speakers. Finally the formula for the selection of one signee as the correct signee is

$$
\hat{s}=\arg \max _{s=1,2 \cdots S} p\left(X \mid \lambda_{s}\right)
$$

and assuming independence among the feature vectors, we get

$$
\hat{s}=\arg \max _{s=1,2 \cdots S} \sum_{i=1}^{T} \log p\left(x_{t} \mid \lambda_{s}\right)
$$

in which $p\left(x_{t} \mid \lambda_{s}\right)$ is given by Eq. (1).

\section{On-line Handwriting Features for GMM}

In this section we describe two different types of features, which can be extracted from signature data and which could be the basis for a GMM.

\subsection{Spatial and Pen Movement Data and Features}

The first kind of handwriting features are sample points of the pen movement while signing. These are in our case 5 -tuples $\left(x_{t}, y_{t}, p_{t}, \theta_{t}, v_{t}\right)$, where $x_{t}, y_{t}$ and $p_{t}$ are the pen tip position coordinates and the pressure, respectively; $\theta_{t}$ is the direction of the stroke tangent in the point $\left(x_{t}, y_{t}\right)$ and $v_{t}$ is the velocity; $t$ is the timeindex. This approach was firstly presented in [1].

\subsection{Spectral Features for Signature Recognition}

Another kind of feature data, which can be extracted from handwriting signals are features from the frequency domain. This is motivated from the domain of speech processing where often cepstral coefficients of speech segments are used. 
The signature can be characterized as a random process This is true because no two signatures of the same person are exactly alike. The same is true for the signals $x_{t}, y_{t}, p_{t}, \theta_{t}$ and $v_{t}$. Having a repeated signal (i.e. periodic) in the time domain, in the frequency domain we have harmonics related to the period of the signal. What we need is the envelope of these harmonics, this characterizes the signature, namely the signee.

The procedure firstly is to take the peaks of the harmonics in the frequency domain. Secondly, in order to take the envelope, we compute the discrete cosine transform of the peaks. So, for each signal $\left(x_{t}, y_{t}, p_{t}, \ldots\right)$ we produce one feature vector.

\section{Acknowledgements}

This work has been supported by the EU Network of Excellence SIMILAR (Proposal Reference Number: FP6507609). The contents of this publication are the sole responsibility of the authors and can in no way be taken to reflect the views of the EU.

\section{References}

1. Richiardi, J., Drygajlo, A.: 'Gaussian Mixture Models for On-line Signature Verification', in Proceedings of WBMA'03, 115-122, 2003.

2. Reynolds, D. A.: A Gaussian Mixture Modeling Approach to Text-Independent Speaker, PhD Thesis, Georgia Institute of Technology, August 1992. 